\title{
Estimador Robusto Recursivo para Sistemas Singulares Incertos com Atraso Invariante no Estado via Método de Elevação *
}

\author{
Elizandra K. Odorico* Kaio D. T. Rocha* Marco H. Terra* \\ * Departamento de Engenharia Elétrica e de Computação, Escola de \\ Engenharia de São Carlos, Universidade de São Paulo, SP, (e-mails: \\ elizandra.odorico@usp.br,kaiodouglas@usp.breterra@sc.usp.br).
}

\begin{abstract}
This paper proposes a recursive solution to the robust estimation problem of uncertain discrete-time singular systems with time-invariant state delay. The proposed approach is based on modeling the singular system with delay as an augmented singular system. A recursive robust estimator is obtained by application of the robust regularized least-squares approach, which combines penalty functions and the optimal solution to the regularized leastsquares problem with uncertainties. A numerical example illustrates the performance of the proposed strategy when compared to an estimator without delay.

Resumo: Este artigo propõe uma solução recursiva para o problema de estimação robusta de sistemas singulares incertos de tempo discreto com atraso invariante no tempo. A abordagem proposta é baseada na modelagem do sistema singular com atraso como um sistema singular aumentado. Um estimador robusto recursivo é obtido pela aplicação da abordagem de mínimos quadrados regularizados robustos, que combina o método de funções penalidade com a solução ótima do problema de mínimos quadrados regularizados com incertezas. Um exemplo numérico ilustra o desempenho da estratégia proposta quando comparado a um estimador sem atraso.
\end{abstract}

Keywords: Delay systems; uncertain systems; discrete-time singular systems; robust regularized least-squares; robust estimation.

Palavras-chaves: Sistemas com atraso; sistemas incertos; sistemas singulares de tempo discreto; mínimos quadrados regularizados robustos; estimativa robusta.

\section{INTRODUÇÃO}

Sistema singular (SS) é uma representação generalizada de sistemas em espaço de estado, amplamente aplicada a sistemas econômicos, redes de comunicação, processos químicos, sistemas biológicos, entre outros (Xu e Lam, 2006). Por outro lado, os sistemas com atraso no estado constituem uma classe de sistemas cuja dinâmica futura depende dos estados atual e passado. Esta classe está presente em diversas aplicações, como sistemas em rede (Liu et al., 2014), problemas de atraso na transferência de informações e risco de perda de dados (Zhang et al., 2013), propagação de epidemias (Zuo et al., 2015) e em sistemas de veículos aéreos não tripulados (Li et al., 2016). O atraso pode afetar consideravelmente as características da planta, alterando sua dinâmica e aumentando a dificuldade na análise de estabilidade e no projeto de estimadores.

A classe de sistemas singulares com atraso no estado (SSAE) é aquela que engloba os dois tipos de sistemas acima mencionados. No entanto, é possível representar essa classe como um sistema singular aumentado através

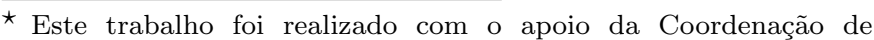
Aperfeiçoamento de Pessoal de Nível Superior (CAPES) e Fundação de Amparo à Pesquisa do Estado de São Paulo (FAPESP) sob protocolo 17/16346-4 vinculado ao Instituto Nacional de Ciência e Tecnologia (INCT) 14/50851-0.
}

da aplicação do método de elevação (do inglês, lifting method), veja, por exemplo, Fridman (2014) e Bortolin et al. (2018). Tal método consiste em um processo de aumento do vetor de estado, transformando o sistema com atraso em um sistema aumentado livre de atraso. Dessa forma, a análise e a síntese de sistemas singulares com atraso invariante são equivalentes àquelas para os sistemas singulares aumentados.

Na literatura, as principais formulações de estimadores para sistemas singulares com atraso no estado são propostas de estimadores dinâmicos, baseados em desigualdades matriciais lineares (LMIs, do inglês, Linear Matrix Inequalities), como os resultados de Kim (2010) e Hu et al. (2013). Além disso, o problema de estimação se destaca nas aplicações de sistemas singulares em rede, onde as perdas de pacotes e os atrasos de comunicação estão presentes (veja, por exemplo, Wang e Sun (2017), Xu et al. (2017)). No melhor conhecimento dos autores, abordagens recursivas de estimadores para o SSAE não foram tratadas na literatura.

Neste artigo, propõe-se uma solução para o problema do estimador robusto recursivo (ERR) para sistemas singulares com atraso invariante no estado. A proposta é inspirada nas abordagens de Fridman (2014) e Bortolin et al. (2018), que utilizaram o método de elevação para representar o sistema com atraso como um sistema aumentado. Além 
disso, neste trabalho é considerado o sistema singular de tempo discreto mais geral, isto é, na forma singular retangular, com ruídos de estado e medida correlacionados e com atraso no estado.

Uma abordagem determinística é utilizada para o desenvolvimento do ERR. Assim, o estimador é projetado em termos de equações de Riccati, através da aplicação de uma técnica de estimativa mínima regularizada robusta desenvolvida por Bianco et al. (2008), que combina o método de função penalidade e mínimos quadrados regularizados com incertezas. O estimador é apresentado na forma de um arranjo matricial simétrico conveniente. A principal característica do ERR é a recursividade do algoritmo dado em termos de uma equação de Riccati aumentada, adequado para aplicações online.

As seções deste artigo estão organizadas da seguinte maneira: na Seção 2, a transformação do SSAE em um SS e o problema de estimação são formulados. Na Seção 3, a técnica de estimativa mínima regularizada robusta é apresentada. Na seção 4, é deduzido o estimador recursivo robusto para SS. Na seção 5, um exemplo numérico ilustra a estratégia proposta, comparando-a com um estimador sem atraso em sua formulação. Na seção 6, as conclusões deste trabalho são apresentadas.

Notações: Seja $\mathbb{R}$ o conjunto de números reais, $\mathbb{R}^{n}$ o conjunto de vetores $n$-dimensionais com elementos em $\mathbb{R}$ e $\mathbb{R}^{m \times n}$ o conjunto de matrizes $m \times n$ reais. $I_{n}$ é a matriz de identidade de dimensão $n \times n$. A matriz nula de dimensão apropriada é denotada por $O$. Para uma matriz real $P$, $P \succ 0(P \succeq 0)$ significa que $P$ é uma matriz simétrica positiva (semi) definida. O símbolo sobrescrito $T$ denota a transposição de matriz. $\mathbb{E}\{x\}$ é o valor esperado de $x$. A norma euclidiana quadrática ponderada de $x$ é denotada por $\|x\|_{P}^{2}=x^{T} P x$.

\section{FORMULAÇÃO DO PROBLEMA}

Considere a classe de sistema singular de tempo discreto com atraso invariante no estado descrito por

$$
\begin{aligned}
\mathcal{E}_{k+1} x_{k+1} & =\mathcal{F}_{k} x_{k}+\mathcal{F}_{d, k} x_{k-d}+\mathcal{G}_{k} \nu_{k}, \\
y_{k} & =\mathcal{H}_{k} x_{k}+\mathcal{K}_{k} \nu_{k}, k \in[0, N], \\
x_{k} & =\varphi_{0}(k), k \in[-d, 0],
\end{aligned}
$$

com

$$
\begin{gathered}
\mathcal{E}_{k+1} \leftarrow E_{k+1}+\delta E_{k+1}, \mathcal{F}_{k} \leftarrow F_{k}+\delta F_{k}, \mathcal{F}_{d, k} \leftarrow F_{d, k}+\delta F_{d, k}, \\
\mathcal{G}_{k} \leftarrow G_{k}+\delta G_{k}, \mathcal{H}_{k} \leftarrow H_{k}+\delta H_{k} \text { e } \mathcal{K}_{k} \leftarrow K_{k}+\delta K_{k},
\end{gathered}
$$

onde

$$
\left[\begin{array}{c}
\mathcal{G}_{k} \\
\mathcal{K}_{k}
\end{array}\right]=\left[\begin{array}{ll}
G_{w, k}+\delta G_{w, k} & G_{v, k}+\delta G_{v, k} \\
K_{w, k}+\delta K_{w, k} & K_{v, k}+\delta K_{v, k}
\end{array}\right] \text { e } \nu_{k}=\left[\begin{array}{c}
w_{k} \\
v_{k}
\end{array}\right],
$$

em que $x_{k} \in \mathbb{R}^{n}$ é o estado no instante $k, x_{k-d} \in \mathbb{R}^{n}$ é o estado atrasado de $d$ amostras e $y_{k} \in \mathbb{R}^{r}$ é a medida. O atraso $d$ é um inteiro positivo constante e $\varphi_{0}(k)$ denota a condição inicial para $k=-d,-d+1, \ldots, 0$. Os vetores $w_{k} \in \mathbb{R}^{p}$ e $v_{k} \in \mathbb{R}^{q}$ são sinais de ruído Gaussianos correlacionados com média zero e matrizes de covariância $Q_{k} \in \mathbb{R}^{p \times p}$ e $R_{k} \in \mathbb{R}^{q \times q}$, respectivamente, e matriz de covariância de termos cruzados $S_{k} \in \mathbb{R}^{p \times q}$, tal que

$$
\mathbb{E}\left\{\left[\begin{array}{c}
w_{k} \\
v_{k}
\end{array}\right]\left[\begin{array}{c}
w_{j} \\
v_{j}
\end{array}\right]^{T}\right\}=\left[\begin{array}{ll}
Q_{k} & S_{k} \\
S_{k}^{T} & R_{k}
\end{array}\right] \delta_{k j}
$$

$\operatorname{com} \delta_{k j}=1$, se $k=j$ e $\delta_{k j}=0$, caso contrário. As matrizes retangulares $E_{k+1}, F_{k}, F_{d, k} \in \mathbb{R}^{m \times n}, G_{w, k} \in \mathbb{R}^{m \times p}, G_{v, k} \in$ $\mathbb{R}^{m \times q}, H_{k} \in \mathbb{R}^{r \times n}, K_{w, k} \in \mathbb{R}^{r \times p}$ e $K_{v, k} \in \mathbb{R}^{r \times q}$ são conhecidas, e as matrizes de incertezas $\delta E_{k+1}, \delta F_{k}, \delta F_{d, k}$, $\delta G_{k}, \delta H_{k}$ e $\delta K_{k}$ são limitadas em norma e modeladas como

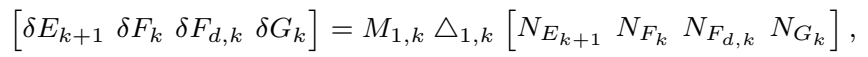

$$
\begin{aligned}
& {\left[\begin{array}{lll}
\delta H_{k} & \delta K_{k}
\end{array}\right]=M_{2, k} \triangle_{2, k}\left[N_{H_{k}} N_{K_{k}}\right] \text {, }}
\end{aligned}
$$

onde as matrizes $M_{1, k} \in \mathbb{R}^{m \times n}$ e $M_{2, k} \in \mathbb{R}^{r \times n}$ são conhecidas e não-nulas, as matrizes $N_{E_{k+1}}, N_{F_{k}}, N_{F_{d, k}} \in$ $\mathbb{R}^{m \times n}, N_{G_{k}} \in \mathbb{R}^{m \times(p+q)}, N_{H_{k}} \in \mathbb{R}^{r \times n}$ e $N_{K_{k}} \in \mathbb{R}^{r \times(p+q)}$ são conhecidas e $\triangle_{1, k} \in \mathbb{R}^{n \times m}$ e $\triangle_{2, k} \in \mathbb{R}^{n \times r}$ são matrizes de contração, com $\left\|\triangle_{i, k}\right\| \leq 1, \forall i=1,2$.

O objetivo deste trabalho é projetar um estimador de estado para o SSAE (1), assumindo que a saída $y_{k}$ e o atraso $d$ estejam disponíveis a cada instante $k$. Como a sequência de estado $\left\{x_{k}\right\}$ associada ao SSAE (1) não é perfeitamente observada, o problema consiste em determinar a estimativa de estado ótima $\hat{x}_{k+1 \mid k}$ para o estado $x_{k+1}$ do sistema, a partir do conjunto de informações $\mathbf{Y}_{k}$ disponível até o passo $k$,

$$
\mathbf{Y}_{k}=\left\{y_{1}, \ldots, y_{k}, d\right\}
$$

Para este propósito, baseando-se em Bianco et al. (2008), admite-se uma interpretação determinística do problema de estimação, fazendo com que este se torne um problema de ajuste de dados, no qual $w_{k}$ e $v_{k}$ são compreendidos como erros de ajuste. Assim, associado ao SSAE (1), considere a seguinte função custo de $N$ estágios:

$$
J_{N}=\left\|x_{0}-\hat{x}_{0 \mid-1}\right\|_{P_{0 \mid-1}^{-1}}^{2}+\sum_{k=0}^{N}\left\|\nu_{k}\right\|_{\Omega_{k}^{-1}}^{2}
$$

sendo $P_{0 \mid-1} \succ 0$ a matriz de ponderação do erro de estimativa inicial $x_{0}-\hat{x}_{0 \mid-1}$, com $\hat{x}_{0 \mid-1}$ representando a estimativa inicial de $x_{0}$ e $\Omega_{k}=\left[\begin{array}{cc}Q_{k} & S_{k} \\ S_{k}^{T} & R_{k}\end{array}\right] \succ 0$ a matriz de ponderação do erro de ajuste $\nu_{k}$.

\subsection{Equivalência com o Sistema Singular Aumentado}

Uma abordagem utilizada no estudo de sistemas com atraso consiste no aumento do vetor de estado do sistema pela aplicação do método de elevação (Fridman, 2014; Bortolin et al., 2018), de tal forma que seja obtido um sistema sem atraso equivalente. Com base nessa abordagem, o SSAE (1) pode ser reescrito como um SS aumentado livre de atraso:

$$
\begin{aligned}
\mathscr{E}_{k+1} z_{k+1} & =\mathcal{A}_{k} z_{k}+\mathcal{B}_{k} \nu_{k} \\
y_{k} & =\mathcal{C}_{k} z_{k}+\mathcal{D}_{k} \nu_{k}, k \in[0, N],
\end{aligned}
$$

com

$$
\begin{gathered}
\mathscr{E}_{k+1} \leftarrow \mathbb{E}_{k+1}+\delta \mathbb{E}_{k+1}, \mathcal{A}_{k} \leftarrow A_{k}+\delta A_{k}, \mathcal{B}_{k} \leftarrow B_{k}+\delta B_{k}, \\
\mathcal{C}_{k} \leftarrow C_{k}+\delta C_{k} \text { e } \mathcal{D}_{k} \leftarrow D_{k}+\delta D_{k},
\end{gathered}
$$

sendo o estado do sistema aumentado $z_{k}$ e a condição inicial $z_{0}$ dados por

$$
z_{k}=\left[\begin{array}{c}
x_{k} \\
x_{k-1} \\
\vdots \\
x_{k-d+1} \\
x_{k-d}
\end{array}\right] \text { e } z_{0}=\left[\begin{array}{c}
\varphi_{0}(0) \\
\varphi_{0}(-1) \\
\vdots \\
\varphi_{0}(-d+1) \\
\varphi_{0}(-d)
\end{array}\right]
$$

As matrizes aumentadas $\mathbb{E}_{k+1}, \quad \delta \mathbb{E}_{k+1}, A_{k}$ e $\delta A_{k} \in$ $\mathbb{R}^{m_{d} \times n_{d}}, B_{k}$ e $\delta B_{k} \in \mathbb{R}^{m_{d} \times(p+q)}, C_{k}$ e $\delta C_{k} \in \mathbb{R}^{r \times n_{d}}$, e $D_{k}$ 
e $\delta D_{k} \in \mathbb{R}^{r \times(p+q)}$, com $m_{d}=(m+d n)$ e $n_{d}=(n+d n)$, são definidas por:

$$
\begin{aligned}
& \mathbb{E}_{k+1}=\left[\begin{array}{cccc}
E_{k+1} & O & \cdots & O \\
O & I_{n} & \cdots & O \\
\vdots & \vdots & \ddots & \vdots \\
O & O & \cdots & I_{n}
\end{array}\right], \quad \delta \mathbb{E}_{k+1}=\left[\begin{array}{cccc}
\delta E_{k+1} & O & \cdots & O \\
O & O & \cdots & O \\
\vdots & \vdots & \ddots & \vdots \\
O & O & \cdots & O
\end{array}\right], \\
& A_{k}=\left[\begin{array}{cccc}
F_{k} & \cdots & O & F_{d, k} \\
I_{n} & \cdots & O & O \\
\vdots & \ddots & \vdots & \vdots \\
O & \cdots & I_{n} & O
\end{array}\right], \delta A_{k}=\left[\begin{array}{cccc}
\delta F_{k} & \cdots & O & \delta F_{d, k} \\
O & \cdots & O & O \\
\vdots & \ddots & \vdots & \vdots \\
O & \cdots & O & O
\end{array}\right] \text {, } \\
& B_{k}=\left[\begin{array}{cc}
G_{w, k} & G_{v, k} \\
O & O \\
\vdots & \vdots \\
O & O
\end{array}\right], \delta B_{k}=\left[\begin{array}{cc}
\delta G_{w, k} & \delta G_{v, k} \\
O & O \\
\vdots & \vdots \\
O & O
\end{array}\right] \\
& C_{k}=\left[\begin{array}{llll}
H_{k} & O & \cdots & O
\end{array}\right], \quad \delta C_{k}=\left[\begin{array}{llll}
\delta H_{k} & O & \cdots & O
\end{array}\right] \text {, } \\
& D_{k}=\left[\begin{array}{ll}
K_{w, k} & K_{v, k}
\end{array}\right] \text { e } \delta D_{k}=\left[\delta K_{w, k} \delta K_{v, k}\right] \text {. }
\end{aligned}
$$

As matrizes de incertezas paramétricas são modeladas como

$$
\begin{aligned}
& {\left[\begin{array}{lll}
\delta \mathbb{E}_{k+1} & \delta A_{k} & \delta B_{k}
\end{array}\right]=\mathcal{M}_{1, k} \Delta_{1, k}\left[\begin{array}{lll}
N_{\mathbb{E}_{k+1}} & N_{A_{k}} & N_{B_{k}}
\end{array}\right],} \\
& {\left[\begin{array}{ll}
\delta C_{k} & \delta D_{k}
\end{array}\right]=\mathcal{M}_{2, k} \Delta_{2, k}\left[\begin{array}{ll}
N_{C_{k}} & N_{D_{k}}
\end{array}\right],}
\end{aligned}
$$

$\operatorname{com}\left\|\Delta_{i, k}\right\| \leq 1, \forall i=1,2$,

$$
\begin{aligned}
& \mathcal{M}_{1, k}=\left[\begin{array}{lll}
M_{1, k}^{T} & \cdots & O
\end{array}\right]^{T} \in \mathbb{R}^{m_{d} \times n}, \Delta_{1, k}=\triangle_{1, k} \in \mathbb{R}^{n \times m}, \\
& N_{\mathbb{E}_{k+1}}=\left[\begin{array}{lll}
N_{E_{k+1}} & \cdots & O
\end{array}\right], N_{A_{k}}=\left[\begin{array}{llll}
N_{F_{k}} & \cdots & O & N_{F_{d, k}}
\end{array}\right] \in \mathbb{R}^{m \times n_{d}}, \\
& N_{B_{k}}=\left[\begin{array}{ll}
N_{G_{w, k}} & N_{G_{v, k}}
\end{array}\right] \in \mathbb{R}^{m \times(p+q)}, \mathcal{M}_{2, k}=M_{2, k} \in \mathbb{R}^{r \times n}, \\
& \Delta_{2, k}=\triangle_{2, k} \in \mathbb{R}^{n \times r}, N_{C_{k}}=\left[\begin{array}{lll}
N_{H_{k}} & \cdots & O
\end{array}\right] \in \mathbb{R}^{r \times n_{d}},
\end{aligned}
$$

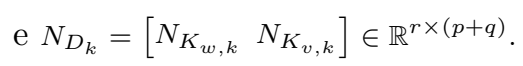

De modo análogo, a versão aumentada da função custo quadrática (2) associada ao SS (3) é dada por

$$
\mathcal{J}_{N}=\left\|z_{0}-\hat{z}_{0 \mid-1}\right\|_{\mathcal{P}_{0 \mid-1}^{-1}}^{2}+\sum_{k=0}^{N}\left\|\nu_{k}\right\|_{\Omega_{k}^{-1}}^{2},
$$

onde $\mathcal{P}_{0 \mid-1} \succ 0$ é a matriz de ponderação do erro de estimativa inicial $z_{0}-\hat{z}_{0 \mid-1}$ e $\Omega_{k} \succ 0$ é a mesma matriz de ponderação de erro de ajuste definida para (2).

Portanto, o SSAE (1) é transformado em um sistema singular aumentado sem a presença de atraso. Em Bianco et al. (2008), o estimador recursivo baseado em equações de Riccati foi estendido para sistemas singulares sujeitos a incertezas paramétricas. Nesta abordagem, um problema de otimização min-max é resolvido, em que a função custo $\mathcal{J}_{N}$ é minimizada sob a máxima influência das incertezas paramétricas $\boldsymbol{\delta}_{k}=\left\{\delta \mathbb{E}_{k+1}, \delta A_{k}, \delta B_{k}, \delta C_{k}, \delta D_{k}\right\}$. Seguindo esta linha, consideramos o seguinte problema de estimação robusta:

$$
\begin{gathered}
\min _{\substack{z_{0}, \ldots, z_{N+1} \\
\nu_{0}, \ldots, \nu_{N}}} \max _{\boldsymbol{\delta}_{0}, \ldots, \boldsymbol{\delta}_{N}} \mathcal{J}_{N} \\
\text { s.a. }\left\{\begin{array}{l}
\mathscr{E}_{k+1} z_{k+1}=\mathcal{A}_{k} z_{k}+\mathcal{B}_{k} \nu_{k} \\
y_{k}=\mathcal{C}_{k} z_{k}+\mathcal{D}_{k} \nu_{k}, k \in[0, N] .
\end{array}\right.
\end{gathered}
$$

Na próxima seção, uma estratégia para a obtenção da sequência ótima $\left\{\hat{z}_{k+1 \mid k}\right\}$ que estima o estado do SS (3) será apresentada.

\section{ESTIMATIVA MÍNIMA REGULARIZADA ROBUSTA}

Esta seção apresenta uma abordagem que fornece um suporte à solução do problema de otimização min-max (7). A solução proposta combina o método de funções penalidade (Luenberger e Ye, 2008; Bazaraa et al., 2006) ao problema de mínimos quadrados regularizados com incertezas nos parâmetros (Sayed, 2001).

Considere o problema de obtenção da estimativa ótima $\hat{z}$ de um vetor de estado $z \in \mathbb{R}^{r}$ a partir de um vetor de observação incerto $(y+\delta y) \in \mathbb{R}^{l}$ proveniente do sistema dinâmico sujeito a incertezas paramétricas

$$
(y+\delta y)=(C+\delta C) z+(D+\delta D) w
$$

sendo $C \in \mathbb{R}^{l \times r}$ e $D \in \mathbb{R}^{l \times p}$ matrizes conhecidas e $w \in \mathbb{R}^{p}$ um vetor de ruído Gaussiano. As matrizes de incertezas paramétricas são modeladas da seguinte forma:

$$
\left[\begin{array}{lll}
\delta C & \delta D & \delta y
\end{array}\right]=L \triangle\left[\begin{array}{lll}
N_{C} & N_{D} & N_{y}
\end{array}\right],\|\triangle\| \leq 1 .
$$

De acordo com Nikoukhah et al. (1992), a estimativa ótima $\hat{z}$ de $z$ é obtida a partir da solução do seguinte problema de otimização min-max determinístico:

$$
\begin{gathered}
\min _{w} \max _{\delta y, \delta C, \delta D} F(z, w)=\|w\|_{U}^{2} \\
\text { s.a. }(y+\delta y)=(C+\delta C) z+(D+\delta D) w,
\end{gathered}
$$

sendo $U \succ 0$ a matriz de ponderação do ruído $w$.

O problema de otimização restrito (8) pode ser transformado em um problema irrestrito equivalente pela aplicação do método de função penalidade, de forma que a restrição seja inserida na função objetivo através do parâmetro de penalidade $\mu>0$, que penaliza qualquer violação desta restrição. Assim, para cada $\mu$, o problema (8) pode ser tratado pelo seguinte problema de mínimos quadrados regularizados com incertezas paramétricas:

$$
\begin{gathered}
\min _{x_{\mu}} \max _{\delta A, \delta b} \mathcal{F}\left(x_{\mu}\right)=\left\|x_{\mu}\right\|_{Q}^{2}+\left\|(A+\delta A) x_{\mu}-(b+\delta b)\right\|_{W_{\mu}}^{2} \\
\operatorname{com}[\delta A \delta b]=M \Delta\left[\begin{array}{ll}
\delta A & N_{b}
\end{array}\right],
\end{gathered}
$$

onde

$$
\begin{gathered}
Q=\left[\begin{array}{cc}
U & O \\
O & O
\end{array}\right], x_{\mu}=\left[\begin{array}{c}
w \\
z
\end{array}\right], A=\left[\begin{array}{cc}
D & C
\end{array}\right], \delta A=\left[\begin{array}{ll}
\delta D & \delta C
\end{array}\right], \\
b=y, \delta b=\delta y, W_{\mu}=\mu I_{l}, M=L \\
\Delta=\triangle, N_{A}=\left[\begin{array}{ll}
N_{D} & N_{C}
\end{array}\right] \text { e } N_{b}=N_{y} .
\end{gathered}
$$

O problema (8) é solucionado iterativamente, isto é, para cada $\mu>0$ de uma sequência monotonicamente crescente $\left\{\mu_{k}\right\}$, uma solução ótima $\hat{x}_{\mu}$ é obtida para o problema (9). Segundo Luenberger e Ye (2008), quando $\mu \rightarrow+\infty$ em (9) a solução obtida equivale à solução ótima do problema restrito $(8)$.

O problema de otimização (9) é resolvido em Sayed (2001) e a solução é reescrita em termos de um arranjo matricial simétrico por Bianco et al. (2008), de acordo com o Lema 1. Lema 1. Considere o problema de otimização (9). Suponha que $W_{\mu}$ é positiva definida. Assim, para cada $\mu>0$, a 
estimativa ótima $\hat{x}_{\mu}$ e a matriz de ponderação $P_{\mu}$ do erro de estimação, $x_{\mu}-\hat{x}_{\mu}$, são dadas por:

$$
\left[\begin{array}{ll}
\hat{x}_{\mu} & P_{\mu}
\end{array}\right]=\left[\begin{array}{l}
O \\
O \\
O \\
O \\
O \\
O \\
I_{s}
\end{array}\right]^{T}\left[\begin{array}{rrrrrrr}
-Q & O & O & I_{s} & O & O & O \\
O & -\widehat{W}_{\mu} & O & O & I_{l} & O & O \\
O & O & -\widehat{\lambda} I_{l} & O & O & I_{l} & O \\
I_{s} & O & O & O & O & O & I_{s} \\
O & I_{l} & O & O & O & O & A \\
O & O & I_{l} & O & O & O & N_{A} \\
O & O & O & I_{s} & A^{T} & N_{A}^{T} & O
\end{array}\right]^{-1}\left[\begin{array}{cc}
O & O \\
O & O \\
O & O \\
O & O \\
b & O \\
N_{b} & O \\
O & -I_{s}
\end{array}\right],
$$

onde $s=p+r$,

$$
\widehat{W}_{\mu}=\left(W_{\mu}^{-1}-\widehat{\lambda}^{-1} M M^{T}\right)^{-1}
$$

e $\widehat{\lambda}=(1+\alpha)\left\|M^{T} W_{\mu} M\right\|$, para algum $\alpha>0$.

Prova. Segue da solução obtida em Sayed (2001), que é reescrita de modo equivalente em termos de um arranjo matricial. A invertibilidade da matriz central em (10) é garantida de acordo com Nikoukhah et al. (1992, Lema 2.1).

\section{ESTIMADOR ROBUSTO RECURSIVO PARA SSAE}

Esta seção apresenta o ERR para sistemas singulares incertos com atraso invariante no estado, isto é, fornece uma solução para o problema de otimização (7) proposto. Este problema é definido com uma função custo de $N$ estágios. Tais funções podem ser tratadas por meio da técnica de programação dinâmica, mais especificamente em sua versão "para frente" (do inglês, forward), que se destaca em problemas de estimação de estado (Larson e Peschon, 1966). Com base nesta abordagem, o problema é resolvido em passos, portanto, divide-se a função custo (6) em várias funções custo de um passo. Assim, a cada passo $k$, consideramos o seguinte problema:

$$
\min _{z_{k+1}, \nu_{k}} \max _{\boldsymbol{\delta}_{k}} \mathcal{J}_{k, \mu},
$$

para $k=0, \ldots, N$, sendo a função custo quadrática dada por

$$
\begin{aligned}
& \partial_{k, \mu}=\left[\begin{array}{c}
z_{k}-\hat{z}_{k \mid k-1} \\
\nu_{k} \\
z_{k+1}
\end{array}\right]^{T}\left[\begin{array}{ccc}
\mathcal{P}_{k \mid k-1}^{-1} & O & O \\
O & \Omega_{k}^{-1} & O \\
O & O & O
\end{array}\right]\left[\begin{array}{c}
z_{k}-\hat{z}_{k \mid k-1} \\
\nu_{k} \\
z_{k+1}
\end{array}\right]+ \\
& \left(\left[\begin{array}{ccc}
\mathcal{A}_{k} & \mathcal{B}_{k} & -\mathscr{E}_{k+1} \\
\mathcal{C}_{k} & \mathcal{D}_{k} & O
\end{array}\right]\left[\begin{array}{c}
z_{k}-\hat{z}_{k \mid k-1} \\
\nu_{k} \\
z_{k+1}
\end{array}\right]-\left[\begin{array}{c}
-\mathcal{A}_{k} \hat{z}_{k \mid k-1} \\
y_{k}-\mathcal{C}_{k} \hat{z}_{k \mid k-1}
\end{array}\right]\right)^{T} \mu I(\bullet),
\end{aligned}
$$

com $\mu>0$ fixado. Observe que o problema de otimização irrestrito (11) é uma aproximação do problema de otimização restrito (7), para cada passo $k$, obtido pelo método de função penalidade. Além disso, (11) é um caso particular do problema (9) quando são realizadas as seguintes identificações:

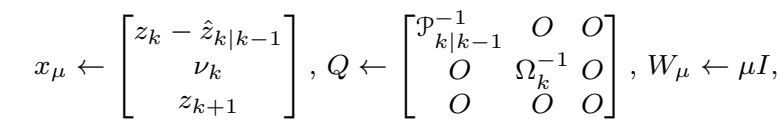

$$
\begin{gathered}
A \leftarrow\left[\begin{array}{ccc}
A_{k} & B_{k} & -\mathbb{E}_{k+1} \\
C_{k} & D_{k} & O
\end{array}\right], b \leftarrow\left[\begin{array}{c}
-A_{k} \hat{z}_{k \mid k-1} \\
y_{k}-C_{k} \hat{z}_{k \mid k-1}
\end{array}\right], M \leftarrow\left[\begin{array}{cc}
\mathcal{M}_{1, k} & O \\
O & \mathcal{M}_{2, k}
\end{array}\right], \\
\delta A \leftarrow\left[\begin{array}{ccc}
\delta A_{k} & \delta B_{k} & -\delta \mathbb{E}_{k+1} \\
\delta C_{k} & \delta D_{k} & O
\end{array}\right], \delta b \leftarrow\left[\begin{array}{c}
-\delta A_{k} \\
-\delta C_{k}
\end{array}\right] \hat{z}_{k \mid k-1}, \Delta \leftarrow\left[\begin{array}{cc}
\Delta_{1, k} & O \\
O & \Delta_{2, k}
\end{array}\right], \\
N_{A} \leftarrow\left[\begin{array}{ccc}
N_{A_{k}} & N_{B_{k}} & -N_{\mathbb{E}_{k+1}} \\
N_{C_{k}} & N_{D_{k}} & O
\end{array}\right] \text { e } N_{b} \leftarrow\left[\begin{array}{l}
-N_{A_{k}} \\
-N_{C_{k}}
\end{array}\right] \hat{z}_{k \mid k-1} .
\end{gathered}
$$

Lema 2. Considere o problema de otimização (11) com $\mu>0$ fixado. A estimativa preditiva ótima $\hat{z}_{k+1 \mid k}$ e a matriz de ponderação $\mathcal{P}_{k+1 \mid k}$ do erro de estimação, $z_{k+1}-$ $\hat{z}_{k+1 \mid k}$, são dados pelo arranjo matricial simétrico (12), para $k \in[0, N]$.

Observação 1. O parâmetro $\widehat{\lambda}$ é definido no intervalo $\left(\left\|\mu \operatorname{diag}\left(\mathcal{M}_{1, k}^{T} \mathcal{M}_{1, k}, \mathcal{M}_{2, k}^{T} \mathcal{M}_{2, k}\right)\right\|, \infty\right)$ para cada $\mu \in(0, \infty)$. Desta forma, a solução ótima no Lema 2 é obtida quando $\mu \rightarrow \infty$. Consequentemente, $\widehat{\lambda}^{-1} \rightarrow 0$ e $\mu^{-1} I \rightarrow 0$. Neste caso, note que a matriz $\left[\mathscr{M}_{k} \mathscr{A}_{k}\right]$ deve ter posto linha pleno, tal que o bloco matricial central em (12) seja invertível. O Algoritmo 1 resume o estimador robusto recursivo proposto para SSAE.

\section{Algoritmo 1 Estimador Robusto Recursivo para SSAE}

Modelo: Assuma o SSAE (1).

Inicialização: Sejam $d, \mu, \hat{\lambda}, N, \hat{z}_{0 \mid-1}$ e $\mathcal{P}_{0 \mid-1} \succ 0$.

Para cada $k=0, \ldots, N$ :

- Definir as matrizes $\mathscr{E}_{k+1}, \mathcal{A}_{k}, \mathcal{B}_{k}, \mathcal{C}_{k}$ e $\mathcal{D}_{k}$ de acordo com (4) e (5).

- Calcular $\hat{z}_{k+1 \mid k}$ e $\mathcal{P}_{k+1 \mid k}$ via (12).

- Extrair $\hat{x}_{k+1 \mid k}$ do vetor de estado aumentado $\hat{z}_{k+1 \mid k}$.

$$
\left[\hat{z}_{k+1 \mid k} \mathcal{P}_{k+1 \mid k}\right]_{\mu}=\left[\begin{array}{l}
O \\
O \\
O \\
O \\
O \\
O \\
O \\
O \\
\widehat{I}
\end{array}\right]^{T}\left[\begin{array}{cccccccccc}
-\mathscr{P}_{k} & I_{\bar{n}_{d}} & O & O & O & O & O & O & O \\
I_{\bar{n}_{d}} & O & O & O & O & O & O & O & I_{\bar{n}_{d}} \\
O & O & \mu^{-1} I_{\bar{m}_{d}} & O & O & O & O & \mathscr{M}_{k} & \mathscr{A}_{k} \\
O & O & O & \widehat{\lambda}^{-1} I_{\bar{m}} & O & O & I_{\bar{m}} & O & O \\
O & O & O & O & -\widehat{\lambda}^{-1} I_{\bar{n}} & O & O & I_{\bar{n}} & O \\
O & O & O & O & O & O & I_{\bar{m}} & O & \mathscr{N}_{\mathscr{A}_{k}} \\
O & O & O & I_{\bar{m}} & O & I_{\bar{m}} & O & O & O \\
O & O & \mathscr{M}_{k}^{T} & O & I_{\bar{n}} & O & O & O & O \\
O & I_{\bar{n}_{d}} & \mathscr{A}_{k}^{T} & O & O & \mathscr{N}_{\mathscr{A}_{k}}^{T} & O & O & O
\end{array}\right]^{-1}\left[\begin{array}{ccc}
O & O \\
z_{k \mid k-1} & O \\
y_{k} & O \\
O & O \\
O & O \\
O & O \\
O & O \\
O & O \\
O & -\widehat{I}
\end{array}\right]
$$

com $n_{d}=n+d n, m_{d}=m+d n, \bar{n}_{d}=2 n_{d}+p+q, \bar{m}_{d}=m_{d}+r, \bar{m}=m+r, \bar{n}=2 n$,

$$
\begin{gathered}
\mathscr{P}_{k}=\left[\begin{array}{ccc}
\mathcal{P}_{k \mid k-1}^{-1} & O & O \\
O & \Omega_{k}^{-1} & O \\
O & O & O
\end{array}\right], \mathscr{M}_{k}=\left[\begin{array}{cc}
\mathcal{M}_{1, k} & O \\
O & \mathcal{M}_{2, k}
\end{array}\right], \mathscr{A}_{k}=\left[\begin{array}{ccc}
A_{k} & B_{k} & -\mathbb{E}_{k+1} \\
C_{k} & D_{k} & O
\end{array}\right], \mathscr{N}_{\mathscr{A}_{k}}=\left[\begin{array}{ccc}
N_{A_{k}} & N_{B_{k}} & -N_{\mathbb{E}_{k+1}} \\
N_{C_{k}} & N_{D_{k}} & O
\end{array}\right], z_{k \mid k-1}=\left[\begin{array}{c}
\hat{z}_{k \mid k-1} \\
O \\
O
\end{array}\right], y_{k}=\left[\begin{array}{c}
O \\
y_{k}
\end{array}\right], \\
\widehat{I}=\left[\begin{array}{lll}
O & O & I_{n_{d}}
\end{array}\right]^{T} \mathrm{e} \hat{\lambda}=(1+\alpha)\left\|\mu \mathscr{M}_{k}^{T} \mathscr{M}_{k}\right\|, \text { para algum } \alpha>0 .
\end{gathered}
$$




\section{EXEMPLO NUMÉRICO}

Considere as matrizes de parâmetros do SSAE (1):

$$
\begin{gathered}
E_{k+1}=\left[\begin{array}{cc}
1 & 0 \\
0 & 1 \\
2 & 0,7
\end{array}\right], F_{k}=\left[\begin{array}{cc}
0,3 & 0 \\
0 & 0,2 \\
0,34 & 0,21
\end{array}\right], G_{v, k}=\left[\begin{array}{l}
1 \\
1 \\
2,7
\end{array}\right], \\
F_{d, k}=\left[\begin{array}{cc}
-1,5 & 0 \\
-1 & -0,5 \\
-0,5 & -0,4
\end{array}\right], G_{w, k}=\left[\begin{array}{cc}
0,4 & 0,1 \\
0,1 & 6 \\
0,87 & 0,62
\end{array}\right], \\
H_{k}=\left[\begin{array}{ll}
1,4 & 0,8]
\end{array}\right], K_{w, k}=\left[\begin{array}{ll}
1,4 & 1,4
\end{array}\right] \\
\text { e } K_{v, k}=1, \forall k \in[0, N],
\end{gathered}
$$

e a condição inicial $x_{k}=\varphi_{0}(k)=[22]^{T}$, para $k \in[-d, 0]$. As matrizes que modelam as incertezas paramétricas são

$$
\begin{gathered}
M_{1, k}=\left[\begin{array}{cc}
0,5 & 0 \\
0 & 0,5 \\
0,01 & 0
\end{array}\right], M_{2, k}=[1,21,2], \\
N_{E_{k+1}}=\left[\begin{array}{ll}
1 & 0 \\
0 & 1 \\
0 & 0
\end{array}\right], N_{F_{k}}=\left[\begin{array}{cc}
0,5 & 0 \\
0 & 0,5 \\
0,5 & 0
\end{array}\right], N_{F_{d, k}}=\left[\begin{array}{cc}
0,3 & 0 \\
0,3 & 0,3 \\
0,3 & 0,3
\end{array}\right], \\
N_{G_{w, k}}=\left[\begin{array}{cc}
0 & 0 \\
0 & 0,05 \\
0 & 0
\end{array}\right], N_{G_{v, k}}=\left[\begin{array}{l}
1 \\
1 \\
1
\end{array}\right], N_{H_{k}}=\left[\begin{array}{ll}
2 & 28,18
\end{array}\right], \\
N_{K_{w, k}}=\left[\begin{array}{ll}
1 & 1
\end{array}\right] \text { e } N_{K_{v, k}}=1, \forall k \in[0, N] .
\end{gathered}
$$

Com relação à função custo de $N$ estágios (2), assumem-se as seguintes matrizes de ponderação:

$$
\begin{gathered}
P_{0 \mid-1}=\left[\begin{array}{ll}
1 & 0 \\
0 & 1
\end{array}\right], Q_{k}=\left[\begin{array}{cc}
7 & 0,0002 \\
0,0002 & 0,001
\end{array}\right], \\
R_{k}=1 \text { e } S_{k}=\left[\begin{array}{c}
0 \\
0,01
\end{array}\right], \forall k \in[0, N] .
\end{gathered}
$$

Para avaliar o desempenho do ERR para sistemas singulares incertos com atraso invariante no estado, será apresentada uma comparação com um estimador robusto que não incorpora a presença de atraso de estado em sua formulação, proposto em Bianco et al. (2008, Teorema 3.1). Todas as rotinas foram implementadas no software MATLAB ${ }^{\circledR}$, versão 9.4 (R2018a).

Para a inicialização do Algoritmo 1, adotam-se

$$
\begin{gathered}
\mu=10^{3}, N=100, \mathcal{P}_{0 \mid-1}=I_{n_{d}}, \widehat{\lambda}=1,5\left\|\mu \mathscr{M}_{k}^{T} \mathscr{M}_{k}\right\| \\
\text { e } \hat{x}_{k \mid k-1}=\left[\begin{array}{ll}
1 & 1
\end{array}\right]^{T} \text { para } k \in[-d, 0] .
\end{gathered}
$$

As Figuras 1 e 2 mostram a média da norma quadrática do erro de estimação em cada passo $k$ para dois cenários de atraso no estado: $d=1$ e $d=10$, respectivamente, considerando $T=1000$ experimentos de Monte Carlo, sendo as médias das normas definidas por

$$
\mathbb{E}\left\{\left\|x_{k}-\hat{x}_{k \mid k-1}\right\|^{2}\right\} \approx \frac{1}{T} \sum_{j=1}^{T}\left\|x_{k}^{(j)}-\hat{x}_{k \mid k-1}^{(j)}\right\|^{2}, \forall k \in[0, N],
$$

onde $x_{k}^{(j)}$ é o estado real no passo $k$ e $\hat{x}_{k \mid k-1}^{(j)}$ é o estado estimado no passo $k$, ambos no experimento $j$. Além disso, os sinais de ruído $w_{k}$ e $v_{k}$ são selecionados aleatoriamente de acordo com suas matrizes de covariância em cada passo de tempo $k$. A cada experimento $j$, as matrizes de

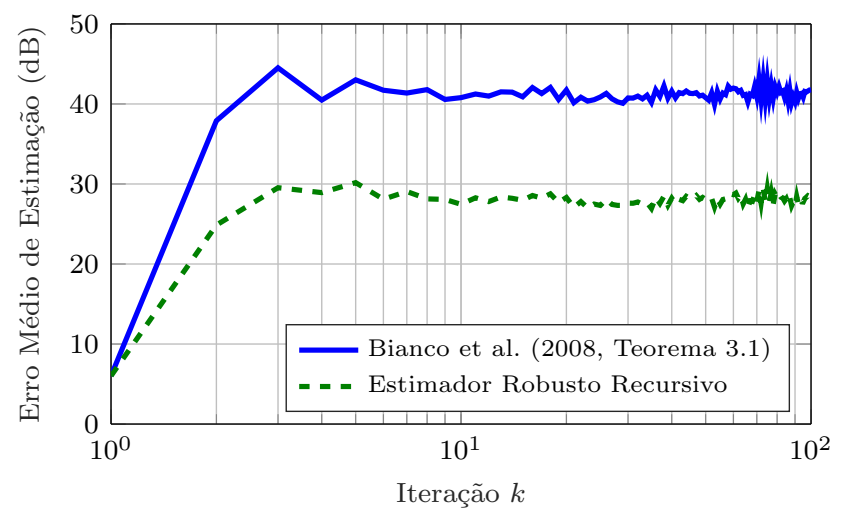

Figura 1. Comparação dos erros médios de estimação quando $d=1$.

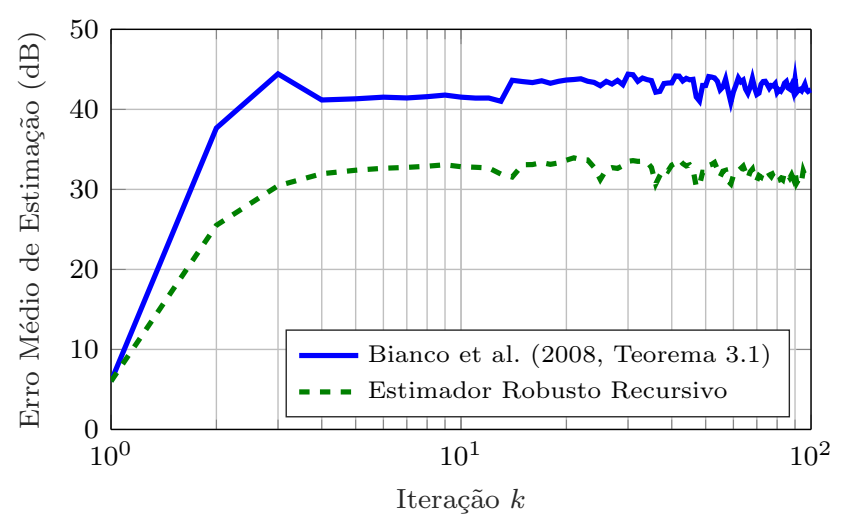

Figura 2. Comparação dos erros médios de estimação quando $d=10$.

contração $\Delta_{i, k}$, com $\left\|\Delta_{i, k}\right\| \leq 1$, para $i=1,2$, são também geradas aleatoriamente.

Observe que o estimador robusto recursivo proposto apresentou erros menores quando comparado ao estimador robusto que não considera atraso de estado, para os dois cenários de atraso. Além disso, pode-se notar que os dois estimadores tiveram seus desempenhos afetados pela variação no atraso $d$, porém, o ERR ainda apresenta um erro menor do que a abordagem de Bianco et al. (2008).

Os valores médios dos erros de estimação nas últimas 50 iterações para os estimadores em cada cenário de atraso de estado são apresentados na Tabela 1. Valida-se, então, a vantagem oferecida pelo estimador robusto recursivo proposto sobre o estimador robusto que não considera atraso em sua formulação, apesar do aumento no valor do atraso $d$.

Tabela 1. Valor médio dos erros de estimação

\begin{tabular}{ccc}
\hline \multirow{2}{*}{ Atrasos } & \multicolumn{2}{c}{ Estimadores } \\
\cline { 2 - 3 } & ERR & Bianco et al. (2008, Teorema 3.1) \\
\hline \hline$d=1$ & 28,1460 & 41,3485 \\
$d=10$ & 31,8592 & 42,8431 \\
\hline
\end{tabular}

As Figuras 3 e 4 mostram as variáveis de estado reais $x_{1, k}$ e $x_{2, k}$ e as estimadas $\hat{x}_{1, k}$ e $\hat{x}_{2, k}$, as quais foram obtidas usando o estimador robusto recursivo para o cenário de 
atraso com $d=1$ durante um dos experimentos. Pode-se considerar que o ERR alcança um bom desempenho de rastreamento.

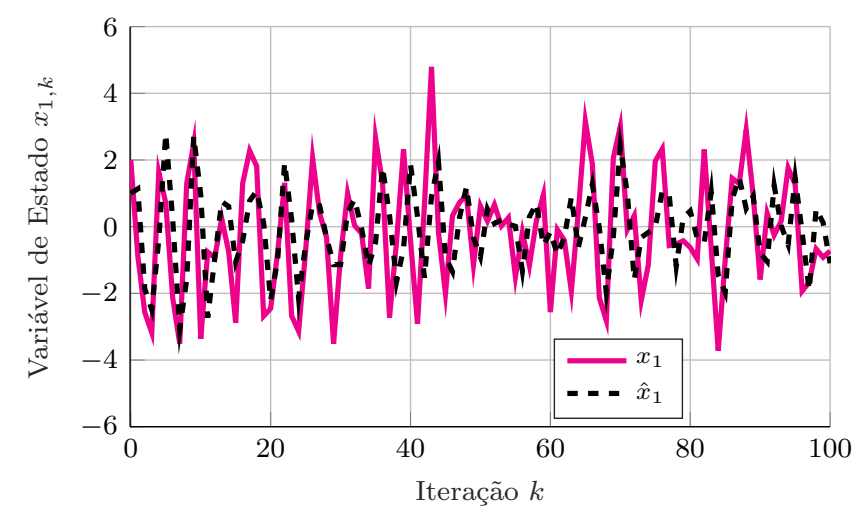

Figura 3. Variável de estado real $x_{1, k}$ e estimada $\hat{x}_{1, k}$ em um experimento com $d=1$.

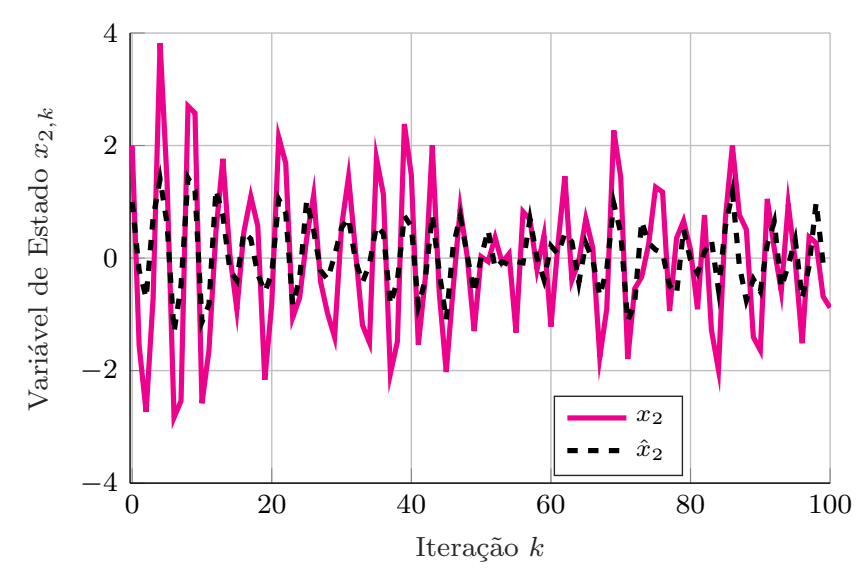

Figura 4. Variável de estado real $x_{2, k}$ e estimada $\hat{x}_{2, k}$ em um experimento com $d=1$.

\section{CONCLUSÃO}

Neste trabalho, uma solução robusta recursiva para o problema de estimação preditora de sistemas singulares de tempo discreto com atraso invariante no estado e incertezas paramétricas foi proposta. Por meio da aplicação do método de elevação, o SSAE foi reescrito como um SS aumentado. Posteriormente, uma metodologia foi proposta para projetar um estimador recursivo robusto para o SS aumentado, apresentado na forma de um arranjo matricial simétrico, que é adequado para aplicações online.

Os resultados da aplicação do ERR em um exemplo numérico demonstraram sua eficácia quando comparado a uma abordagem que não incorpora a presença de atraso em sua formulação. No entanto, o método de elevação aumenta a dimensão do sistema dinâmico, o que pode exigir um elevado esforço computacional. O desenvolvimento de métodos recursivos alternativos capazes de amenizar o aumento da dimensão do estado causado pelo atraso será considerado em trabalhos futuros.

\section{REFERÊNCIAS}

Bazaraa, M.S., Sherali, H.D., e Shetty, C.M. (2006). Nonlinear Programming: Theory and Algorithms. Wiley, Hoboken, $3^{\text {a }}$ edição. Capítulo 9.

Bianco, A.F., Ishihara, J.Y., e Terra, M.H. (2008). Optimal robust prediction for general discrete time singular systems. In 10th International Conference on Control, Automation, Robotics and Vision, 1793-1798.

Bortolin, D.C., Odorico, E.K., e Terra, M.H. (2018). Robust linear quadratic regulator for uncertain linear discrete-time systems with delay in the states: an augmented system approach. In IEEE European Control Conference (ECC), 1578-1583.

Fridman, E. (2014). Introduction to Time-Delay Systems: Analysis and Control. Birkhäuser Basel, $1^{\mathrm{a}}$ edição.

Hu, Z., Zhu, H., e Zhao, J. (2013). Further results on $H_{\infty}$ filtering for a class of discrete-time singular systems with interval time-varying delay. Circuits, Systems, and Signal Processing, 32(3), 1081-1095.

Kim, J.H. (2010). Delay-dependent robust $H_{\infty}$ filtering for uncertain discrete-time singular systems with interval time-varying delay. Automatica, 46(3), 591-597.

Larson, R. e Peschon, J. (1966). A dynamic programming approach to trajectory estimation. IEEE Transactions on Automatic Control, 11(3), 537-540.

Li, M., Hu, Z., Xie, F., e Fan, D. (2016). Stability of dynamic quantized UAV airborne system with packet losses and time-varying delay. In 15th International Symposium on Parallel and Distributed Computing (ISPDC), 406-411.

Liu, K., Fridman, E., e Hetel, L. (2014). Networked control systems: A time-delay approach. In European Control Conference (ECC), 1434-1439.

Luenberger, D.G. e Ye, Y. (2008). Linear and Nonlinear Programming. Springer, New York, $3^{\mathrm{a}}$ edição. Capítulo 13.

Nikoukhah, R., Willsky, A.S., e Levy, B.C. (1992). Kalman filtering and Riccati equations for descriptor systems. IEEE Transactions on Automatic Control, 37(9), 13251342.

Sayed, A.H. (2001). A framework for state-space estimation with uncertain models. IEEE Transactions on Automatic Control, 46(7), 998-1013.

Wang, X. e Sun, S. (2017). Optimal recursive estimation for networked descriptor systems with packet dropouts, multiplicative noises and correlated noises. Aerospace Science and Technology, 63, 41-53.

Xu, Q., Zhang, Y., e Xiao, S. (2017). Networked $H_{\infty}$ filtering for discrete-time nonlinear singular system with mixed random delays and packet dropouts. In 36th Chinese Control Conference (CCC).

$\mathrm{Xu}$, S. e Lam, J. (2006). Robust control and filtering of singular systems. Lecture Notes in Control and Information Science. Springer, Berlin.

Zhang, L., Gao, H., e Kaynak, O. (2013). Network induced constraints in networked control systems: A survey. IEEE Transactions on Industrial Informatics, 9(1), 403416.

Zuo, L., Liu, M., e Wang, J. (2015). The impact of awareness programs with recruitment and delay on the spread of an epidemic. Mathematical Problems in Engineering, 2015, 1-10. 